\title{
Contribuição farmacêutica na logística de medicamentos e acompanhamento clínico de pacientes com tuberculose e hanseníase na atenção primária à saúde
}

\author{
Pharmaceutical contribution in medication logistics and clinical follow-up of patients with \\ tuberculosis and leprosy in primary health care
}
Contribución farmacéutica en la logística de medicamentos y seguimiento clínico de pacientes con tuberculosis y lepra en atención primaria de salud

Milena Cristina da Silva ${ }^{1 *}$, José Gildo de Lima1.

\begin{abstract}
RESUMO
Objetivo: Relatar uma experiência iniciada no primeiro ano da especialização da Residência Multiprofissional em Saúde da Família, da Universidade Federal de Pernambuco, em uma Unidade de Saúde da Família da cidade do Recife. Relato de experiência: O relato baseia-se na dificuldade que a unidade de saúde tinha em manter o cuidado continuadamente e estabelecer um acompanhamento de saúde mais fidedigno dos pacientes com hanseníase e tuberculose, contendo início e término de tratamento, prescrição medicamentosa e condição clínica dos pacientes, e na inserção do profissional farmacêutico na logística dos medicamentos e acompanhamento clínico dos pacientes de tuberculose e hanseníase juntamente com a equipe de saúde. Considerações finais: Neste contexto, espera-se motivar a equipe de saúde com a estruturação desse processo para que se tenha a percepção da importância do acompanhamento no cuidado desses pacientes e na relevância da inserção do profissional farmacêutico para uma melhor condução dos processos organizativos e clínicos.
\end{abstract}

Palavras-chave: Hanseníase, Tuberculose, Medicamento.

\begin{abstract}
Objective: To report an experience initiated in the first year of the specialization of the Multiprofessional Residence in Family Health, from the Federal University of Pernambuco, in a Family Health Unit in the city of Recife. Experience report: The report is based on the difficulty that the health unit had in maintaining care continuously and establishing a more reliable health monitoring of patients with leprosy and tuberculosis, containing the beginning and end of treatment, drug prescription and clinical condition of the patients, and the insertion of the professional pharmacist in drug logistics and clinical follow-up of tuberculosis and leprosy patients together with the health team. Final considerations: In this context, it is expected to motivate the health team with the structuring of this process so that they have the perception of the importance of monitoring in the care of these patients and the relevance of the insertion of the pharmaceutical professional for a better management of the organizational and clinical processes.
\end{abstract}

Key words: Leprosy, Tuberculosis, Medicine.

\section{RESUMEN}

Objetivo: Informar una experiencia iniciada en el primer año de la especialización de la Residencia Multiprofesional en Salud de la Familia, de la Universidad Federal de Pernambuco, en una Unidad de Salud de la Familia en la ciudad de Recife. Informe de experiencia: El informe se basa la dificultad que tuvo la unidad de salud para mantener más confiable la atención de manera continua y un monitoreo de salud más confiable de los pacientes con lepra y tuberculosis, conteniendo el inicio y el final del tratamiento, la prescripción de medicamentos y el estado clínico de los pacientes, y la inserción del profesional farmacéutico en la logística de los pacientes. medicación y seguimiento clínico de los pacientes con tuberculosis y lepra

1 Universidade Federal de Pernambuco, Recife - PE. *E-mail: milenaa_cris@hotmail.com 
junto con el equipo de salud. Consideraciones finales: En este contexto, se espera motivar al equipo de salud con la estructuración de este proceso para que tengan la percepción de la importancia del seguimiento en la atención de estos pacientes y la relevancia de la inserción del profesional farmacéutico para una mejor gestión de los procesos organizativos y clínicos.

Palabras clave: Lepra, Tuberculosis, Medicamento.

\section{INTRODUÇÃO}

As Doenças de Notificações Compulsórias (DNCs), são doenças que necessitam ser monitorizadas e comunicadas às autoridades de saúde, pois podem causar surtos e epidemias. Portanto, requer medidas eficazes para a prevenção e controle, sendo fundamental para impedir a disseminação da doença e o aparecimento de novos casos. Atrelada a essa lista de doenças, existe um sistema de informação de agravos de notificação (SINAN) que tem como objetivo coletar informações para subsidiar o processo de investigação (SILVA GA e OLIVEIRA CMG, 2014).

A hanseníase, considerada uma doença compulsória, ainda é um problema de saúde pública, apesar da prevalência ter reduzido nos últimos anos. O Brasil vem registrando cerca de33.000 casos por ano, sendo o segundo país com a maior quantidade de casos do mundo. É considerado um agravo subnotificado e apresenta elevado risco de adoecimento na cidade do Recife. A taxa de detecção foi de 58,7 casos por 100 mil habitantes em 2007 e passou para 29,2 em 2017 (RECIFE, 2018).

É uma doença crônica, infectocontagiosa, causada pelo agente etiológico Mycobacterium leprae, bacilo que acomete principalmente olhos, mãos e pés, por ter preferência pela pele e os nervos periféricos com alterações sensitivas (MARINHO FD, et al., 2015).

Esses fatores de comprometimento podem ser responsáveis por sequelas permanentes no indivíduo. Para que isso não ocorra, o diagnóstico precisa ser rápido, com o tratamento adequado e prioridade de acesso aos serviços públicos de saúde para qualquer dificuldade no tratamento (SANTOS AR e IGNOTTI E, 2020). Portanto, a hanseníase é uma doença curável com tratamento poliquimioterápico, o qual dura de seis a doze meses, a partir de uma classificação utilizada paucibacilares ou multibacilares referente ao número de lesões, a carga do bacilo e ao nível de comprometimento dos nervos periféricos do paciente (RIBEIRO GC e LANA FCF, 2015).

Além da hanseníase existe outra doença compulsória que precisa ser referida: a tuberculose. O Brasil faz parte do grupo dos 22 países que concentram $80 \%$ dos casos de tuberculose no mundo, ocupando a 16. ${ }^{\text {a }}$ posição em número absoluto de casos. Entre 2007 e 2017, registrou-se uma média anual de 1550 casos novos de tuberculose no Recife (RECIFE, 2018). A tuberculose é uma doença infecciosa e transmissível, causada pelo Mycobacterium tuberculosis, que afeta principalmente os pulmões, mas pode ocorrer em outros órgãos e sistemas (BRASIL, 2019).

A tuberculose é transmitida por via aérea a partir da aspiração de partículas contendo o bacilo cujo é eliminado pela tosse, fala ou espirro do indivíduo que a contraiu e que esteja com a doença ativa (TEIXEIRA $A Q$, et al., 2020). O tratamento tem duração de seis meses, se não apresentar nenhum tipo de intercorrências, como retratamento, reingresso após abandono ou uma recidiva após cura (BARREIRA D, 2018).

O tratamento de infecção latente por tuberculose (ILTB) ocorre quando uma pessoa se encontra infectada pelo bacilo, Mycobacterium tuberculosis mas não possui manifestações da doença ativa. Portanto é extremamente importante realizar atendimento clinico e exames nos contactantes dos pacientes com a doença ativa (BRASIL, 2018a).

O tratamento medicamentoso tanto da hanseníase quanto da tuberculose perpassa por um ciclo da assistência farmacêutica que abrange a seleção, programação, aquisição, armazenamento, distribuição e dispensação de medicamentos, além do acompanhamento, avaliação e supervisão das ações. Tais medicamentos são utilizados para o tratamento de agravos específicos, agudos ou crônicos que integram o componente estratégico da assistência farmacêutica (BRASIL, 2011). 
Nesse processo logístico da assistência farmacêutica, é essencial a participação do farmacêutico para estruturar o fluxo dos medicamentos, realizar a dispensação e orientações ao usuário (SILVESTRE CC, et al., 2017).

O estudo tem o objetivo de aproximar os profissionais de saúde da unidade (Médicos, enfermeiros, dentistas e agente de saúde) no cuidado e acompanhamento aos pacientes de tuberculose e hanseníase priorizando esse atendimento e articulando com os demais serviços para que o paciente consiga efetivamente realizar a conclusão dos tratamentos. Além disso demostrar a importância do farmacêutico está inserido junto a equipe nessas praticas do cuidado pois, é um profissional essencial tanto na logística dos medicamentos como nos atendimentos clínicos.

\section{RELATO DE EXPERIÊNCIA}

Estudo descritivo do tipo relato de experiência, fundamentado em pesquisas de artigos científicos que abordam assuntos pertinentes ao tema proposto. Foi realizado durante o curso da Residência Multiprofissional em Saúde da Família da Universidade Federal de Pernambuco, entre os meses de abril de 2019 e julho de 2020, com os casos de tuberculose e hanseníase em uma Unidade de Saúde da Família.

Para o acompanhamento clínico desses pacientes e a logística dos medicamentos, no primeiro momento, foi preciso organizar o processo, conhecer os pacientes e as equipes de saúde correspondentes. A partir disso, foi elaborada uma planilha com dados pessoais e dos medicamentos para monitoramento. Em outro momento, os pacientes começaram a receber os medicamentos e recebiam atendimento clínico para dúvidas e queixas.

Embora a unidade possua uma farmácia, as atividades desenvolvidas referentes à logística dos medicamentos como dispensação, avaliação de estoque e falta do medicamento não estão sendo desenvolvidas atualmente pelo farmacêutico, pois o mesmo não possui vínculo para essa atividade. $\mathrm{Na}$ realidade, esse profissional na unidade tem como competência realizar atendimentos clínicos individuais, compartilhados com outros profissionais, e educação em saúde junto com a equipe Núcleo Ampliado à Saúde da Família e atenção básica (NASF- AB), que tem como objetivo ampliar as ações e serviços com uma abordagem integral ao indivíduo no aspecto social, familiar e cultural, organizando as práticas de saúde através da integração das ações de promoção, prevenção e reabilitação.

Observou-se na unidade que o farmacêutico não tem participação na logística de distribuição e controle dos medicamentos sendo fundamental também ao processo de saúde dos usuários.

No período deste estudo, os profissionais estavam realizando um curso de atenção básica no qual eles traziam situações abordadas no curso para serem discutidas na unidade, e um dos questionamentos foi em relação à ausência de controle e acompanhamento dos medicamentos dos pacientes com tuberculose e hanseníase, pois naquele momento não tinha um profissional responsável para tal situação. A partir disso percebemos a possibilidade de inserir o farmacêutico em uma atividade inerente à sua atribuição e, também, de aproximação desse profissional à equipe de saúde. Desse modo, ao demonstrarmos interesse nessa atividade, o controle e dispensação mensal dos medicamentos de tuberculose e hanseníase ficaram sob nossa responsabilidade.

Por conseguinte, algumas perguntas começaram a surgir: Quem são esses pacientes? Quais são as equipes de saúde? Como organizar esse processo? Os agentes de saúde começaram então a colaborar na organização. Foram descobertos quem eram os pacientes e a equipe de saúde correspondente; foi elaborada uma tabela de acompanhamento com nome do paciente, equipe de saúde, número do SUS, medicamento, período de tratamento, assinatura e data de recebimento, e coletados os contatos dos pacientes para informações e acompanhamento.

Os medicamentos são provenientes da farmácia a qual é responsável por enviar os mesmos para as unidades. Sendo assim, começamos a pensar em uma forma estratégica para tratar as faltas, mudanças e suspensões dos tratamentos, para evitar que ficassem sobras do medicamento na unidade ou que não chegasse o medicamento na data correspondente ao início do tratamento. 
Durante esse processo, conhecemos cada um dos pacientes e as dinâmicas de vida, pois isso é primordial para que se consiga fazer saúde. A partir daí, atendemos os pacientes mensalmente para a entrega dos medicamentos e realizamos atendimentos referentes às dúvidas, queixas ou algum tipo de necessidade que tivesse no momento que podia ter relação a questões psicológicas, sociais e nutricionais.

Posteriormente, foram levadas essas demandas para reunião do NASF-AB, realizada semanalmente, para que outros profissionais pudessem contribuir nas questões individuais de cada um. E no caso de alguma queixa de saúde repassava para equipe a necessidade do médico realizar atendimento.

Mensalmente, no caso de chegar a data de entrega dos medicamentos e algum dos usuários não tivesse ido buscar, realizávamos uma busca ativa para compreender o motivo do ocorrido e oportunamente lembrávamos aos pacientes da importância da continuidade do tratamento para a própria saúde e das pessoas contactantes. Nessas ocasiões, percebemos que eles se sentiam surpresos e bem cuidados.

Evidentemente, ainda que mínimas, houve algumas desistências ao longo desse processo, porque infelizmente alguns usuários possuíam fatores que dificultavam a continuidade do tratamento, alguns eram alcoolistas, outros não tinham moradia, renda, mas com todas essas questões foram mínimas as desistências. Então, começamos a ter a percepção de que a partir dessa organização os profissionais da unidade começaram a se aproximar e se integrar um pouco mais com os pacientes e também demonstrar a importância a que essas doenças necessitam.

Concomitantemente a esse processo, nos deparamos com uma urgência sanitária: a COVID-19. E todos juntos profissionais de saúde, preceptores, residentes, tutores e graduandos pensamos em uma maneira de informar a população sobre essa doença e os cuidados necessários. Assim, foi elaborado um projeto de extensão intitulado "Enfrentamento ao COVID-19: produzir saúde e defender a vida".

Um dos eixos do projeto foi assistência à saúde, em que participamos na elaboração de vídeos explicativos direcionados para o público em geral, e particularmente às pessoas acometidas com hanseníase e tuberculose, com a finalidade de transmitir de forma mais precisa os cuidados que esses pacientes deveriam ter em relação à COVID-19.

Indubitavelmente, foi uma realização pessoal e profissional muito grande saber que, mesmo minimamente conseguimos contribuir com essas pessoas que, às vezes, não são vistas devido ao preconceito que está atrelado a esses agravos, tanto dos profissionais quanto da população em geral, demonstrar para os profissionais a importância de o farmacêutico estar inserido nas conduções relacionadas aos medicamentos, como também na condição clínica do paciente. Precisamos desmistificar essas doenças e fazer saúde sem discriminar fortalecendo vínculos e acesso para a população.

\section{DISCUSSÃO}

A tuberculose e a hanseníase são doenças que há décadas existem mesmo havendo medicamento e tratamento ainda apresentam grandes números de infectados e por isso precisam ser priorizadas. A Estratégia de Saúde da Família, na atenção primária, é a porta de entrada para o início e acompanhamento do tratamento dessas enfermidades, onde o acesso a essa população e a construção de vínculo precisa ser construída, pois sabe-se da dificuldade em tratar essas enfermidades, sendo importante também a corresponsabilização do usuário no processo de saúde (MACEDO VLM, et al., 2019). Esse fato corrobora como estudo de Nogueira JÁ, et al. (2012), que retrata a importância do vínculo para estabelecer laços interpessoais que refletem na cooperação mútua entre usuários e profissionais de saúde.

É notório perceber que após a vinculação construída pelo profissional farmacêutico com os pacientes da unidade acometidos pelas doenças houve um baixo índice de abandono no tratamento, sendo realizado dezoito atendimentos para tuberculose e sete para hanseníase, com apenas 2 abandonos referente a tuberculose. Demonstra-se com isso, a obtenção de um maior controle em relação ao período de tratamento e a situação clínica do paciente. Em outro estudo, reafirma-se a importância do papel do farmacêutico para o sucesso do tratamento dessas doenças (KOTWISKI FO, 2018). 
Também foi observado em um estudo sobre as principais causas do abandono ao tratamento relacionadas diretamente ao paciente consistindo, em $58,3 \%$ dos casos, o uso de drogas ilícitas, o uso de bebidas alcoólicas e a falta de dinheiro. Isso ratifica as questões trazidas nesse relato demostrando as dificuldades sociais que permeiam tal população (SÁ AMM, et al., 2017).

É importante ressaltar que o indivíduo precisa ser visto integralmente não por partes que, às vezes, não estão realizando o tratamento, por questões sociais de renda, moradia, ou também por danos psicológicos trazidos pela descriminação sofrida até hoje atrelada por essas doenças. Com isso se dá a importância da inserção multiprofissional nesses atendimentos. Dessa forma, no estudo realizado por Finotti RFC, et al. (2020) verificou-se em um serviço de referência para hanseníase que em $43,1 \%$ dos atendimentos, os pacientes possuíam sintomas depressivos tendo como consequência o aumento do uso de drogas psicoativas.

Por conseguinte, o trabalho realizado de forma multiprofissional garante um atendimento mais integral e resolutivo ao usuário tendo, assim, o propósito de uma melhor intervenção no ambiente em que atuam. Para Araújo MBS e Rocha PM (2007), a influência acerca dos diversos aspectos que compõem o processo de saúde-doença é uma consequência do trabalho em equipe.

A atuação do farmacêutico na atenção básica perpassa tanto por funções gerenciais como também assistenciais. A primeira refere-se às atividades relacionadas à logística do medicamento que está ligado também à prescrição e a dispensação, já a segunda visa o cuidado ao usuário com ações direcionadas ao paciente, não ao medicamento, de forma a garantir o uso correto dos mesmos e alcançar resultados terapêuticos satisfatórios (COSTA EA, et al., 2017).

É relevante evidenciar que a prática referente à logística do medicamento não está sendo desenvolvida pelo farmacêutico na unidade de saúde, contudo faz parte da sua atribuição (COSTA KS, et al., 2017).

Vale salientar que o Ministério da Saúde, por meio da portaria no 154 de 24 de janeiro de 2008, criou os Núcleos de Apoio a Saúde da Família e Atenção Básica (NASF-AB), o qual revela as atividades do farmacêutico na atenção básica, perpassando pela gestão dos medicamentos a atividades de atendimentos clínicos (BRASIL, 2018b).

É notório afirmar que a atenção básica se configura como porta de entrada para as demandas da população, incluindo a identificação e encaminhamento precoce de problemas para outros níveis de complexidade. Desse modo, considerando as situações complexas enfrentadas pelo indivíduo, família e comunidade nos territórios da Estratégia de Saúde da Família, em 2020, a saúde pública se deparou com uma adversidade maior: a pandemia ocasionada pelo novo coronavírus (SOUZA SS, et al., 2021).

Isto posto, a atenção primária precisou elaborar estratégias de remodelamento nos atendimentos ao usuário tanto no espaço físico criando procedimentos de distanciamento e obrigatoriedade do uso de máscaras; quanto no manejo à assistência, realizando telemonitoramentos aos pacientes dos grupos prioritários estabelecido pela a unidade de saúde e acompanhamento dos pacientes positivos ao COVID 19 por telefone, para manter a longitudinalidade do cuidado (RODRIGUES AP, et al., 2020).

É considerável sublinhar a relevância do profissional residente nessa conjuntura frente a uma urgência sanitária que pode contribuir junto a população na realização de materiais educativos, na assistência ao usuário e na extensão desse processo para a universidade para fomentar a construção integral do ensinoserviço-comunidade (LUCENA JF e SENA JGB, 2020).

Conclui-se que as práticas relacionadas a essas doenças precisam ser monitoradas e efetivamente priorizadas com educação em saúde e busca ativa no território, e que a inserção do profissional farmacêutico juntamente com a equipe de saúde nesse processo foi de extrema importância para uma maior vinculação entre os outros profissionais e usuários. Espera-se que o presente estudo contribua para demonstrar 0 potencial da atuação do farmacêutico no processo de saúde-doença e que práticas multiprofissionais possibilitam um atendimento mais qualificado, efetivo e seguro para o paciente. 


\section{REFERÊNCIAS}

1. ARAÚJO MBS, ROCHA PM. Trabalho em equipe: um desafio para a consolidação da estratégia de saúde da família. Ciência \& Saúde Coletiva, 2007; 12(2): 455-464.

2. BARREIRA D. Os desafios para a eliminação da tuberculose no Brasil. Epidemiologia e Serviços de Saúde, 2018; 27(1):e00100009.

3. BRASIL. Assistência Farmacêutica no SUS. Brasília: CONASS, 2011; $186 \mathrm{p}$.

4. BRASIL. Protocolo de vigilância da infecção latente pelo Mycobacterium tuberculosis no Brasil. Brasília: Ministério da Saúde, 2018a; 32 p.

5. BRASIL. Práticas Farmacêuticas no Núcleo Ampliado de Saúde da Família e Atenção Básica (Nasf AB). Brasília: Ministério da Saúde, 2018b; 33 p.

6. BRASIL. Manual de Recomendações para o Controle da Tuberculose no Brasil. Brasília: Ministério da Saúde, 2019; $364 \mathrm{p}$.

7. COSTA EA, et al. Concepções de assistência farmacêutica na atenção primária à saúde, Brasil. Revista de Saúde Pública, 2017; 51 Supl 2:5s.

8. COSTA KS, et al. Avanços e desafios da assistência farmacêutica na atenção primária no Sistema Único de Saúde. Revista de Saúde Pública, 2017; 51 Supl 2: 3s.

9. FINOTTI RFC, et al. Transtornos mentais comuns e fatores associados entre pessoas com hanseníase: análise transversal em Cuiabá, 2018. Epidemiologia e Serviços de Saúde, 2020; 29(4): e2019279.

10. KOTWISKI FO. O papel do farmacêutico na adesão do paciente ao tratamento da hanseníase em dois municípios do interior da Bahia. Trabalho de Conclusão de Curso (Bacharelado em Farmácia) - Faculdade Maria Milza, Governador Mangabeira, 2018; $93 \mathrm{p}$.

11. MACEDO VLM, et al. Avaliação da estratégia saúde da família em São Sebastião - Distrito Federal. Enfermagem em Foco, 2019; 10(2): 15-21.

12. MARINHO FD, et al. Hanseníase em menores de 15 anos: uma revisão bibliográfica. Revista Família, Ciclos de Vida e Saúde no Contexto Social, 2015, 3(2): 1-11.

13. LUCENA JF, SENA JGB. Residência Integrada Multiprofissional em Saúde e a pandemia COVID-19: um relato de experiência. Revista Eletrônica Acervo Saúde, 2020; 12(9): e4964.

14. NOGUEIRA JA, et al. Vínculo e acesso na estratégia saúde da família: percepção de usuários com tuberculose. Revista da Rede de Enfermagem do Nordeste, 2012, 13(4): 784-793.

15. RECIFE. Plano Municipal de Saúde 2018 - 2021. Recife: Secretaria de Saúde do Recife, 2018.

16. RIBEIRO GC, LANA FCF. Incapacidades físicas em hanseníase: caracterização, fatores relacionados e evolução. Revista Cogitare Enfermagem, 2015; 20(3): 496-503.

17. SÁ AMM, et al. Causas de abandono do tratamento entre portadores de tuberculose. Revista da Sociedade Brasileira de Clínica Médica, 2017; 15(3): 155-160.

18. SANTOS AR, IGNOTTI E. Prevenção de incapacidade física por hanseníase no Brasil: análise histórica. Ciência \& Saúde Coletiva, 2020; 25(10): 3731-3744.

19. RODRIGUES AP, et al. Telemonitoramento como estratégia de cuidado longitudinal a grupos prioritários em tempos da COVID-19: uma experiência na atenção primária à saúde do município de Vitória-ES. APS em Revista, 2020; 2(2): 189-196.

20. SILVA GA, OLIVEIRA CMG. O registro das doenças de notificação compulsória: a participação dos profissionais da saúde e da comunidade. Journal of Epidemiology and Infection Control, 2014; 4(3): 215-220.

21. SILVESTRE CC, et al. Planejamento estratégico situacional na farmácia de um hospital universitário: um relato de experiência. Revista Brasileira de Farmácia Hospitalar e Serviços de Saúde, 2017; 8(2): 18-25.

22. SOUZA SS, et al. Influência da cobertura da atenção básica no enfrentamento da COVID-19. Journal Health NPEPS, $2021 ; 6(1): 1-21$.

23. TEIXEIRA AQ, et al. Tuberculose: conhecimento e adesão às medidas profiláticas em indivíduos contatos da cidade do Recife, Pernambuco, Brasil. Cadernos Saúde Coletiva, 2020; 28(1): 116-129. 